\title{
DINÂMICA POPULACIONAL DO SURUBIM-TIGRE, Pseudoplatystoma tigrinum (VALENCIENNES, 1840) NO MÉDIO AMAZONAS (SILURIFORMES, PIMELODIDAE)
}

\author{
Mauro Luis RUFFINO', Victoria Judith ISAAC ${ }^{2}$
}

RESUMO - Dados de comprimento e de captura e esforço da pesca do surubim-tigre (Pseudoplatystoma tigrinum) obtidos durante o periodo de 1993 a 1996, no Médio Amazonas, foram utilizados para descrever as pescarias e estimar as taxas de crescimento e mortalidade da espécie. Os parâmetros populacionais médios estimados foram $\mathrm{L}_{\infty}=180,0 \mathrm{~cm}$ (comprimento total), $\mathrm{W}_{\infty}=56,56 \mathrm{~kg}, \mathrm{k}=0,29 \mathrm{ano}^{-1}, \mathrm{C}=0,9$; WP= Novembro, $\mathrm{M}=0,47$ ano $^{-1}, \mathrm{Z}=1,42 \mathrm{ano}^{-1}, \mathrm{~F}=0,95 \mathrm{ano}^{-1} \mathrm{e}_{\mathrm{c}}=87,0 \mathrm{~cm}$. A análise do rendimento por recruta mostra que o estoque está sendo explorado em seu limite de sustentabilidade.

Palavras-chave: surubim-tigre, Pseudoplatystoma tigrinum, Amazônia, crescimento, mortalidade.

Population Dynamics of Striped Catfish, Pseudoplatystoma tigrinum (Valenciennes, 1840) in the Middle Amazon (Siluriformes, Pimelodidae)

ABSTRACT - Catch and effort data and length data series of striped catfish Pseudoplatystoma tigrinum obtained through 1993 to 1996 in the Lower Amazon, Brazil, were used to describe the fishery and to estimate growth and mortality rates. Mean population parameters estimated were $\mathrm{L}_{x}$ $=180,0 \mathrm{~cm}$ (total length), $\mathrm{W}_{\infty}=56,56 \mathrm{~kg}, \mathrm{k}=0,29$ year $^{-1}, \mathrm{C}=, \mathrm{WP}=$ November, $\mathrm{M}=0,47$ year $^{-1}, \mathrm{Z}=1,42$ year $^{-1}, \mathrm{~F}=0,95$ year $^{-1}$ e $\mathrm{L}_{\mathrm{c}}=87,0 \mathrm{~cm}$. Yield-per-recruit analysis shows that the stocks are being exploited near to limit of sustainability.

Key words: striped catfish, Pseudoplatystoma tigrinum, Amazon, growth, mortality.

\section{INTRODUÇÃO}

\section{O gênero Pseudoplatystoma} Bleecker apresenta três espécies distribuídas na maior parte da América do Sul tropical e subtropical, as quais se destacam por seu grande tamanho e valor comercial na pesca de águas interiores. As espécies $P$. fasciatum e $P$. tigrinum apresentam ampla distribuição nas Bacias Amazônica e do Orinoco (Reid, 1983; Barthem \& Goulding, 1997).

As duas espécies distinguem-se facilmente tanto por suas diferenças na forma da cabeça como pela coloração (Reid, 1983; Ferrreira et al., 1998). De acordo com a descrição de Coy (1994), $P$. tigrinum apresenta barbilhões maxilares curtos que não sobrepassam a cabeça, distinguindo-se de $P$. fasciatum pela presença de uma profunda fontanela que alcança o processo occipital, nadadeira dorsal, anal e caudal com pontos conspícuos. Possui desenho cromático dos lados do corpo, geralmente com bandas verticais interespaçadas com pontos ou manchas, escuro dorsalmente e branco ventralmente, com 16 faixas que se unem. Podem apresentar manchas ou pontos encerrados dentro destes desenhos, todas as nadadeiras radíadas com manchas na forma de pontos.

P. tigrinum, conhecido popularmente como surubim-tigre, pintado ou caparari, é a espécie do gênero que apresenta maior valor comercial na pesca profissional e maior proporção nos desembarques de Santarém. Em 1992,

Projeto IARA (IBAMA/GOPA-GTZ) - Av. Tapajös, 2267 - Cep. 68.040-000 - Santarém, PA 2 Universidade Federal do Pará - Centro de Ciências Biológicas/Dept ${ }^{\circ}$ de Biologia - Campus Universitário do Guamá - Cep. 66.075-900 - Belém, PA 
P. tigrinum foi responsável por $7,2 \%$ das $3.713 \mathrm{t}$ desembarcadas em Santarém, estando em $5^{\circ}$ lugar na composição das espécies (Ruffino \& Isaac, 1994). De acordo com Goulding (1979), $P$. tigrinum era a espécie mais importante explorada nas pescarias dos rios Guaporé e Mamoré, na década de 70. Segundo Payne (1987), 95\% do peixe desembarcado em Trinidad, Bolívia, era composto de 4 espécies, Pseudoplatystoma fasciatum, P. tigrinum, Colossoma macropomum e C. brachypomum. Santos (1987), descrevendo a composição e situação da pesca no estado de Rondônia, cita que em 1984 a produção de $P$. tigrinum foi de $41,2 \%$ do total de 104,5 t desembarcadas em Vila Pimenteira, à margem do rio Guaporé. Petrere (1992) estimou em 48\%, a participação de $P$. tigrinum nas capturas do rio Mamoré entre jan/86 a maio/87.

Apesar da importância comercial, dados sobre a biologia reprodutiva e dinâmica populacional do surubim-tigre da bacia amazônica são escassos. Algumas publicações referem-se a estudos em áreas fora da Amazônia brasileira (Reid, 1983; Payne, 1987; Gil \& Martinez, 1995). Os poucos estudos sobre o surubim-tigre na Amazônia brasileira limitam-se a algumas referências da espécie em trabalhos sobre a pesca comercial (Barthem \& Goulding, 1997; Goulding, 1979, 1980; Petrerre, 1992; Smith, 1981). Ruffino \& Isaac (1995) e Isaac et al. (no prelo) reportam dados preliminares sobre a reprodução da espécie.

O presente trabalho é parte dos resultados do Projeto IARA (IBAMA, 1995) e objetiva estimar os parâmetros populacionais de crescimento e mortalidade de Pseudoplatystoma tigrinum. Com isso, pretende-se fornecer informações necessárias para a adoção de possiveis medidas para o manejo da espécie.

\section{MATERIAL E MÉTODOS}

Os dados para o presente trabalho foram obtidos de amostras selecionadas ao acaso, de surubimtigre desembarcado nos mercados e frigoríficos de Santarém, durante os anos de 1993 a 1996. De cada indivíduo registrou-se o comprimento total, medido da extremidade do focinho à extremidade da nadadeira caudal, com precisão de $1 \mathrm{~cm}$. O peso total foi obtido através de balanças com precisão de $100 \mathrm{~g}$.

Os parâmetros $\mathbf{a}$ e $\mathbf{b}$ da relação $\mathrm{W}=\mathrm{a} \cdot \mathrm{L}^{\mathrm{b}}$ foram estimados após transformação logarítmica pelo método dos mínimos quadrados.

Os parâmetros de crescimento foram estimados com base nas distribuições mensais de freqüência dos comprimentos dos anos 1993 e 1995, agrupadas em classes de $5 \mathrm{~cm}$. Os dados ajustaram-se ao modelo sazonal de von Bertalanffy (Pauly, 1984) através do método Elefan (Pauly \& David, 1981). O índice de performance de crescimento $\left(\phi^{\prime}=\log _{10} \mathrm{k}+2 \log _{10} \mathrm{~L}_{\infty}\right)$ de Pauly \& Munro (1984) foi calculado para comparar os parâmetros de crescimento do surubim-tigre com os valores da literatura.

Estimativas de mortalidade natural (M) foram obtidas da relação empírica de Pauly (1980) e a mortalidade total (Z) foi obtida através da curva de captura convertida para comprimentos (Sparre et al., 1992) e pelo método de Beverton \& 
Holt (1956), a partir dos comprimentos médios na captura. As estimativas do rendimento por recruta foram obtidas através do modelo de Beverton \& Holt (1957). Para a aplicação destes métodos foi utilizado o programa FISAT de Gayanilo et al. (1994).

Para a descrição da pesca, dados de captura e esforço foram obtidos diariamente, através de entrevistas com todos os pescadores e/ou proprietários das embarcações que aportaram na cidade de Santarém, durante os anos de 1993 a 1996, conforme descrito em Isaac \& Ruffino (no prelo).

\section{RESULTADOS}

\section{A Pesca}

A captura de surubim-tigre está correlacionada com o nivel do rio e com as chuvas na região. Em geral, as viagens em que ocorrem maior porcentagem de surubim-tigre ocorrem durante a vazante e seca e as menores porcentagens durante a cheia (Fig. 1), sendo que em média, $22,5 \%$ das viagens capturam surubim-tigre. A produção de surubim-tigre apresentam dois picos, um maior em setembro e outro bem menor em dezembro-janeiro, ambos durante a vazante, seca e inicio da enchente, respectivamente (Fig. 2).

Os desembarques da espécie nos portos de Santarém, para os anos de 1993 a 1996, foram 236, 171, 274 e 182 t, respectivamente (Tab. 1). A produção mensal nesse período variou de um mínimo $1,5 \mathrm{t}$ em abril de 1996 a $86 \mathrm{t} \mathrm{em}$ setembro de 1993, com média de $18 \mathrm{t}$ e desvio padrão de 20 t. Em média a espécie representa $6 \%$ da produção desembarcada na região. Porém se contabilizadas somente as viagens nas quais há ocorrência de surubim-tigre, e que podem ser consideradas como pescarias dirigidas à captura de peixes lisos, essa proporção aumenta para

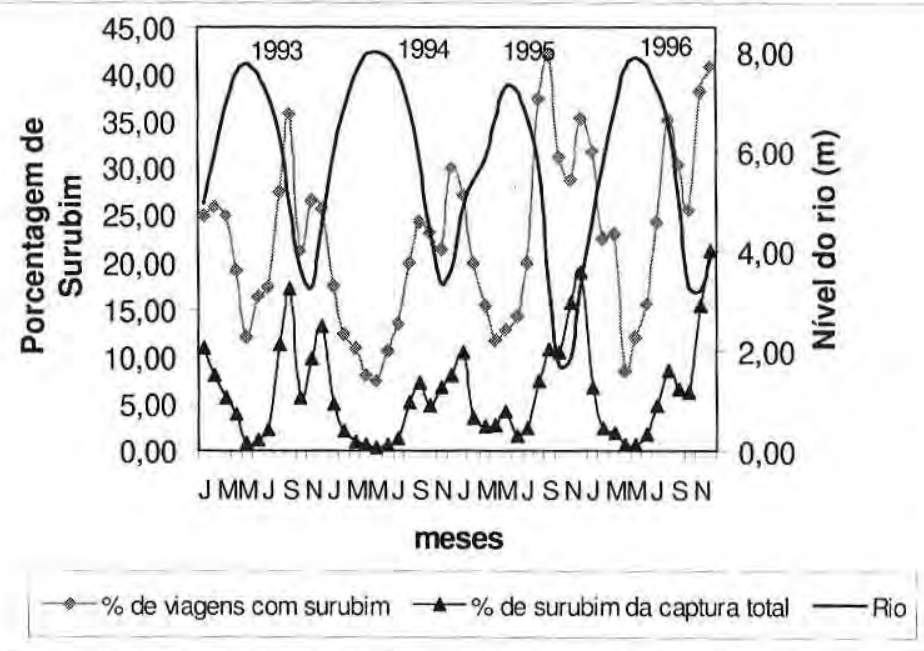

Figura 1. Proporção de viagens nas quais ocorre a captura de surubim-tigre (Pseudoplatystoma tigrimum), composição porcentual de surubim-tigre em relação ao total de capturas e nivel do rio para o periodo de 1993 a 1996 em Santarém. 


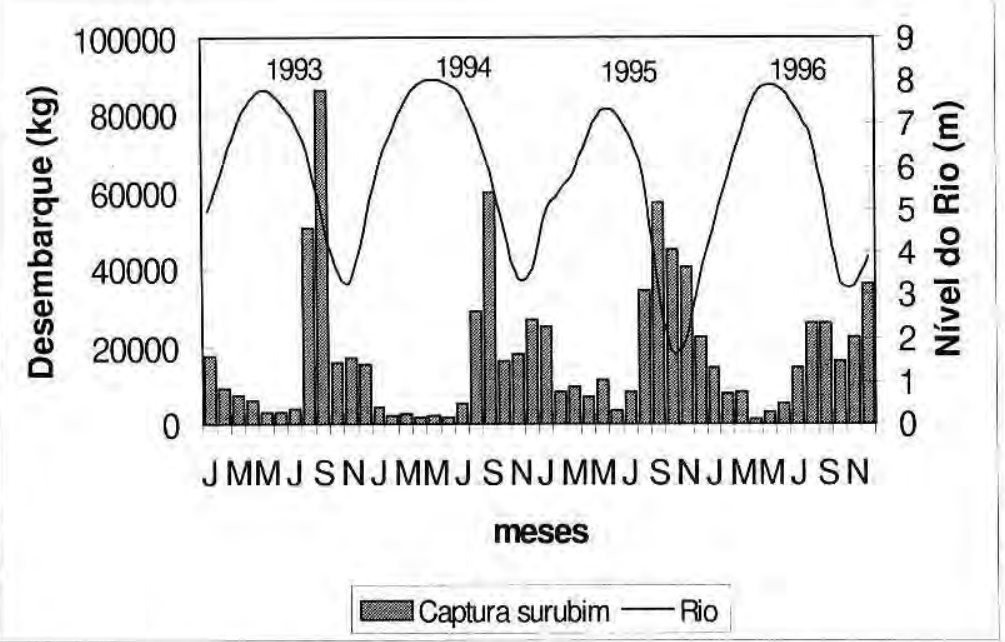

Figura 2. Produção mensal de surubim-tigre (Pseudoplatystoma tigrinum) nos portos de desembarque de Santarém no periodo de 1993 a 1996.

Tabela 1. Captura, comprimento total (Lt) médio de captura e porcentagem de indivíduos abaixo do tamanho minimo permitido de Pseudoplatystoma tigrinum no período de 1993 a 1996 , em Santarém, no Médio Amazonas.

\begin{tabular}{lcccc}
\hline ANO & $\begin{array}{c}\text { Captura } \\
\text { anual }(\mathrm{t})\end{array}$ & $\begin{array}{c}\text { № de indivíduos } \\
\text { amostrados }\end{array}$ & $\begin{array}{c}\text { Comprimento } \\
\text { total médio }(\mathrm{cm})\end{array}$ & $\begin{array}{c}\% \text { de indivíduos abaixo do } \\
\mathrm{L}_{\text {min }} \text { permitido }(80 \mathrm{~cm})\end{array}$ \\
\hline 1993 & 235,7 & 1.690 & 75,5 & 58,9 \\
1994 & 170,8 & 1.043 & 70,1 & 67,1 \\
1995 & 273,6 & 2.576 & 75,9 & 66,1 \\
1996 & 181,9 & 1.189 & 69,8 & 84,9 \\
\hline
\end{tabular}

$26 \%$ do total capturado. A espécie encontra-se entre as 10 espécies mais pescadas no periodo (Tab. 2).

A captura por unidade de esforço (CPUE) para o surubim-tigre, expressa como $\mathrm{kg} /$ pescador/dia, mostra a tendência similar às capturas totais. A produtividade média mensal oscilou entre 1,12 e $8,32 \mathrm{~kg} /$ pescador/dia e foi maior em 1995, quando as chuvas foram menos intensas e menor em 1994 quando a duração do periodo de cheia foi maior em relação aos demais anos (Fig. 3). A estimativa média geral da CPUE ( $\mathrm{kg} /$ pescador/dia) e seu respectivo desvio padrão foi de $3,67 \pm 1,75$.
O surubim-tigre é capturado com uma grande diversidade de aparelhos de pesca. No entanto, a rede de emalhar (ou malhadeira) é responsável por $37,4 \%$ das capturas da região, seguida da bubuia com $29,4 \%$ e mistura de mais de uma arte (por exemplo, rede de emalhar e espinhel) com $25,2 \%$. Os outros $8 \%$ incluem tarrafa, linha, espinhel, zagaia e arpão (Fig. 4). A malhadeira é empregada com intensidade variável durante todo o ano, enquanto que durante o período da safra, entre julho e novembro, outras artes de pesca são empregadas adicionalmente, principalmente bubuia e espinhel. 
Tabela 2. Composição relativa das principais espécies nas capturas das pescarias do Médio Amazonas desembarcadas em Santarém no periodo 1993-1996.

\begin{tabular}{lccc}
\hline Nome científico & Nome vulgar & Captura (t) & $(\%)$ \\
\hline Hypophthalmus spp. & Mapará & 3417 & 24 \\
Brachyplatystoma flavicans & Dourada & 1846 & 13 \\
Prochilodus nigricans & Curimata & 1798 & 12 \\
Semaprochilodus insignis & Jaraqui-escama-grossa & 943 & 6 \\
Plagioscion spp. & Pescada & 886 & 6 \\
Pseudoplatystoma tigrinum & Surubum-tigre & 862 & 6 \\
Brachyplatystoma vaillantii & Piramutaba & 568 & 4 \\
Schizodon fasciatus; Leporinus spp. & Aracu & 557 & 4 \\
Milossoma spp.; Myleus spp.; Metynnis spp. & Pacu & 479 & 3 \\
Colossoma macropomum & Tambaqui & 464 & 3 \\
\hline Outras espécies & & 2785 & 19 \\
\hline
\end{tabular}

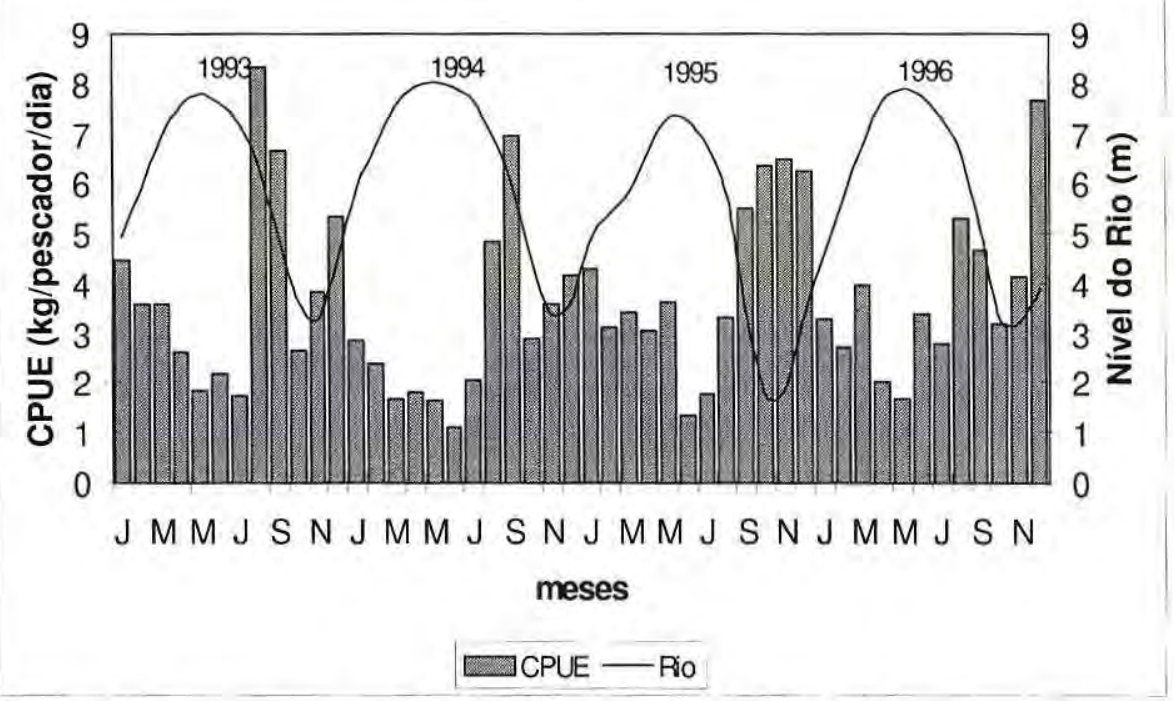

Figura 3. Captura por unidade de esforço $(\mathrm{kg} /$ pescador/dia) para o surubim-tigre (Pseudoplatystoma tigrinum) nas pescarias do Médio Amazonas.

As capturas de surubim-tigre ocorrem em lagos e rios da região, numa proporção quase que eqüitativa, sendo que a pesca concentra-se nos lagos durante de enchente e cheia, e nos rios durante a vazante e seca (Fig. 5).

\section{Relação Peso-Comprimento}

$\mathrm{O}$ ajuste da relação entre o peso e o comprimento do surubim-tigre (Fig. 6) resultou na seguinte equação:

$$
W t=0,0026 \cdot L t^{3,25}
$$

O coeficiente de correlação (r) foi de 0,984 com 1.160 dados e $\mathrm{L}_{\min }=42 \mathrm{~cm} \mathrm{e}$ $\mathrm{L}_{\max }=130 \mathrm{~cm}$. O valor do expoente b é significativamente diferente de 3,0 , de maneira que o crescimento em peso pode ser assumido como alométrico. 


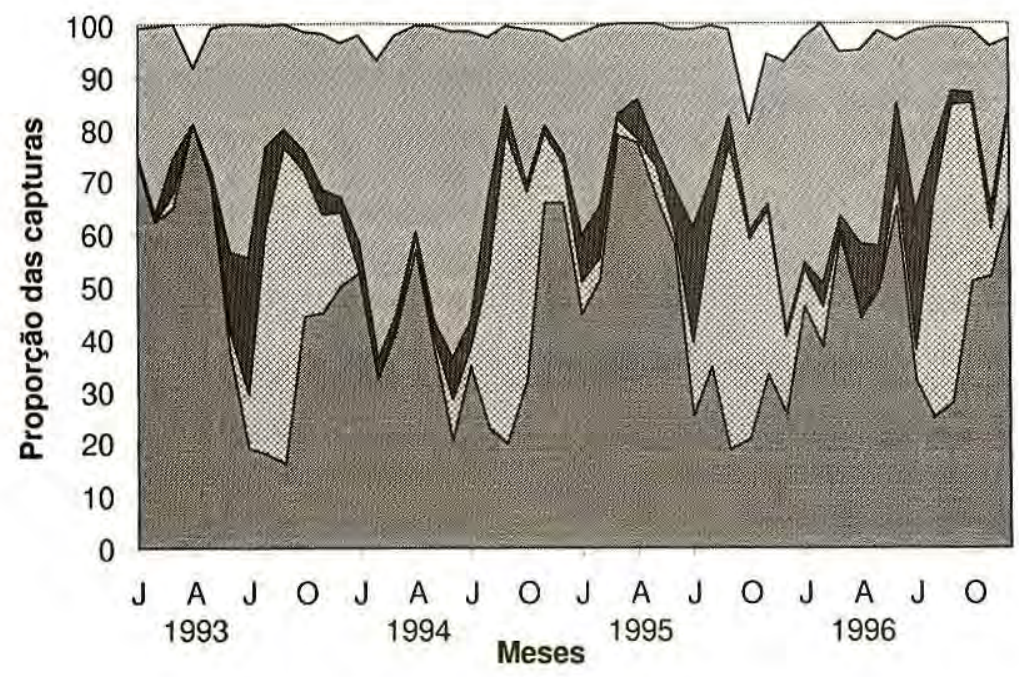

\section{$\square$ Malhadeira $\otimes$ Bubuia $\square$ Espinhel $\llbracket$ Varios $\square$ Outros}

Figura 4. Proporção das capturas de surubim-tigre (Pseudoplatystoma tigrinum) por arte de pesca nas pescarias do Médio Amazonas. A diferença completando $100 \%$ deve-se a dados não informados.

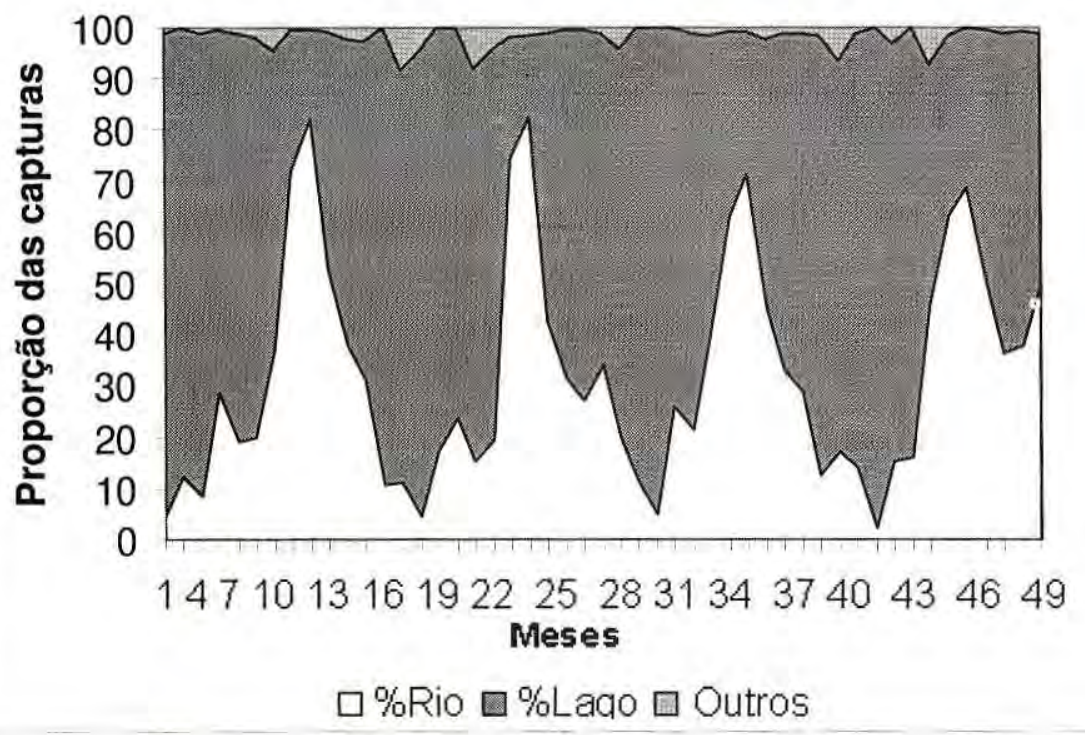

Figura 5. Proporção das capturas de surubim-tigre (Pseudoplatystoma tigrinum) por ambiente nas pescarias do Médio Amazonas. A diferença completando 100\% deve-se a dados não informados. 


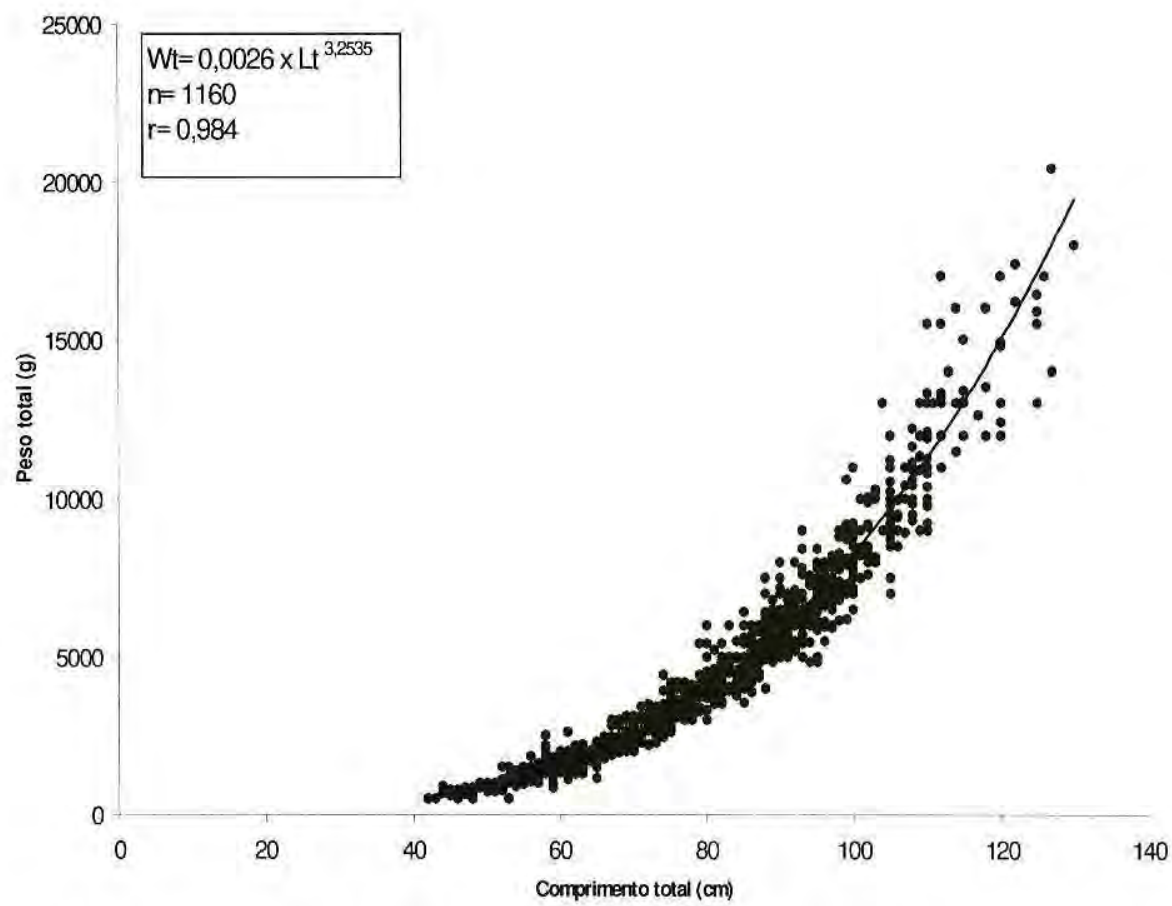

Figura 6. Relação peso-comprimento para surubim-tigre (Pseudoplatystoma tigrinum) da região do Médio Amazonas.

\section{Crescimento em comprimento}

$\mathrm{O}$ surubim-tigre recruta-se à pesca com aproximadamente $30 \mathrm{~cm}$ de comprimento total. Peixes acima de $140 \mathrm{~cm}$ não são freqüentes nas amostras. O comprimento médio, para todos os dados, foi de $75,7 \mathrm{~cm}$ com um desvio padrão de $17,8 \mathrm{~cm}$. Maiores individuos ocorrem quase sempre na metade do ano, próximo do mês de junho, no fim do período das chuvas (Fig. 7). Nota-se que o tamanho médio dos indivíduos tem diminuído e o número de exemplares abaixo do tamanho mínimo permitido $(80 \mathrm{~cm})$ vem aumentando ao longo dos anos, chegando, em 1996, a quase $85 \%$ dos exemplares amostrados (Tab, 1).

Os parâmetros que descrevem o crescimento em comprimento do surubimtigre foram mais ou menos constantes nos dois anos analisados, 1993 e 1995 . As estimativas de $\mathrm{L}_{\infty}$ e k obtidas foram 180 $\mathrm{cm}$ e 0,29 ano $^{-1}$ para ambos anos. Os valores de $\mathrm{C}=0,85$ e WP $=1,0$ ano $^{-1}$ para 1993, e $\mathrm{C}=0,95$ e WP $=0,8$ ano $^{-1}$ para 1995 indicam que o crescimento do surubim-tigre oscila sazonalmente de forma sinuzóide, e que as taxas de crescimento foram menores em dezembro de 1993 e outubro de 1995 , quando o nível da água está baixo, ou seja, durante a seca. Inversamente, as taxas de crescimento foram mais elevadas no meio do ano, durante os meses de cheia. Estimativas do índice do performance de crescimento $\phi$ ' resultaram em 3,97 para ambos anos (Tab. 3). 


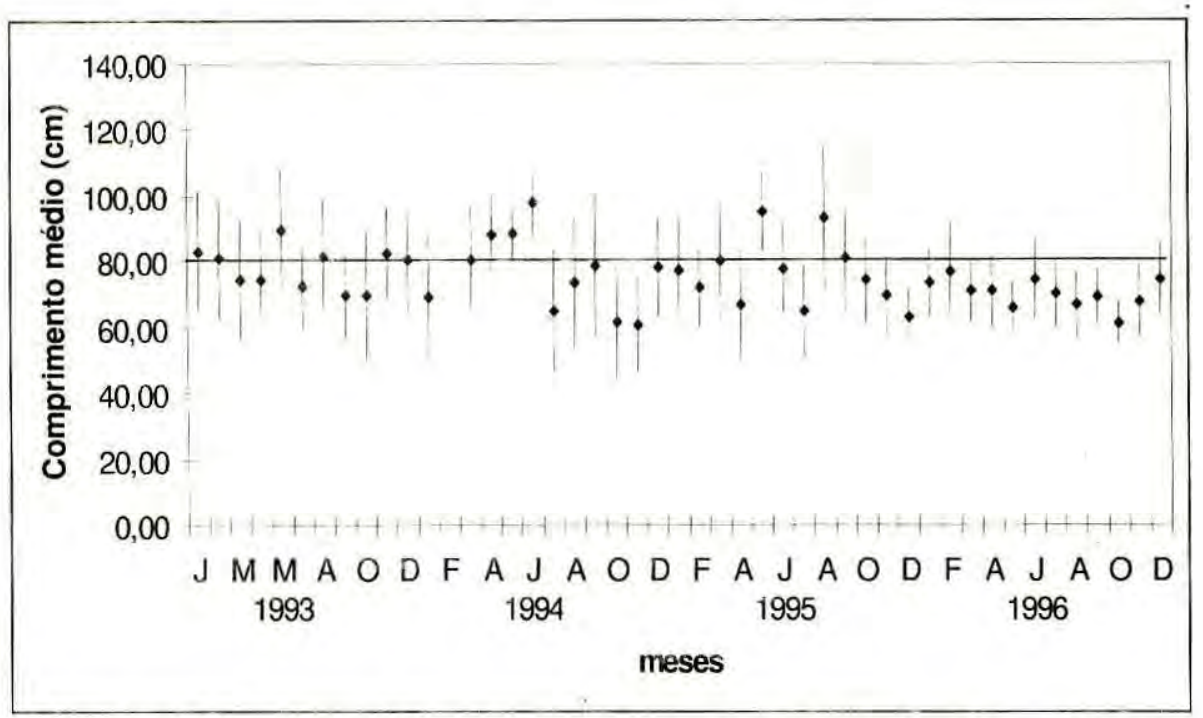

Figura 7. Comprimentos médios mensais \pm um desvio padrão para surubim-tigre (Pseudoplatystoma tigrinum) do Médio Amazonas no periodo de 1993 a 1996 . Nota: $80 \mathrm{~cm}$ é o comprimento mínimo permitido para a captura pela legislação pesqueira vigente.

Tabela 3. Parâmetros da dinâmica populacional do surubim-tigre (Pseudoplatystoma tigrinum) do Médio Amazonas.

\begin{tabular}{lccc}
\hline Parâmetros & 1993 & 1995 & Parâmetros médios \\
\hline K (1/ano) & 0,29 & 0,29 & 0,29 \\
$\mathrm{~L}_{\infty}(\mathrm{cm})$ & 180,00 & 180,00 & 180,00 \\
Wo (kg) & 56,559 & 56,559 & 56,559 \\
$\mathrm{C}$ & 0,85 & 0,95 & 0,90 \\
\hline WP (1/ano) & 1,00 (dezembro) & 0,80 (outubro) & 0,90 (novembro) \\
\hline Rn & 0,157 & 0,184 & - \\
$\phi^{\prime}$ & 3,97 & 3,97 & 3,97 \\
M (1/ano) & 0,47 & 0,47 & 0,47 \\
$Z$ (1/ano) & 1,48 & 1,37 & 1,42 \\
$F(1 / a n o)$ & 1,01 & 0,90 & 0,95 \\
E & 0,68 & 0,66 & 0,67 \\
$L_{c}$ (cm) & 85,00 & 86,60 & 85,8 \\
\hline
\end{tabular}

A figura 8 mostra a curva de crescimento, estimada pelo ELEFAN, superimposta sobre os dados de freqüência dos comprimentos. Os resultados sugerem que $P$. tigrinum tem uma longevidade de, no mínimo, 10 anos. Indicam também que a pesca opera sobre, no mínimo, 5 coortes da população. A tabela 4 mostra uma chave idade-comprimento estimada a partir do modelo de von Bertalanffy e usando os parâmetros médios dos anos de 1993 e 1995. Os comprimentos correspondem à idade relativa, uma vez que o valor real de $t_{0}$ é desconhecido. A idade absoluta não pode ser calculada com base em apenas dados de comprimento. 

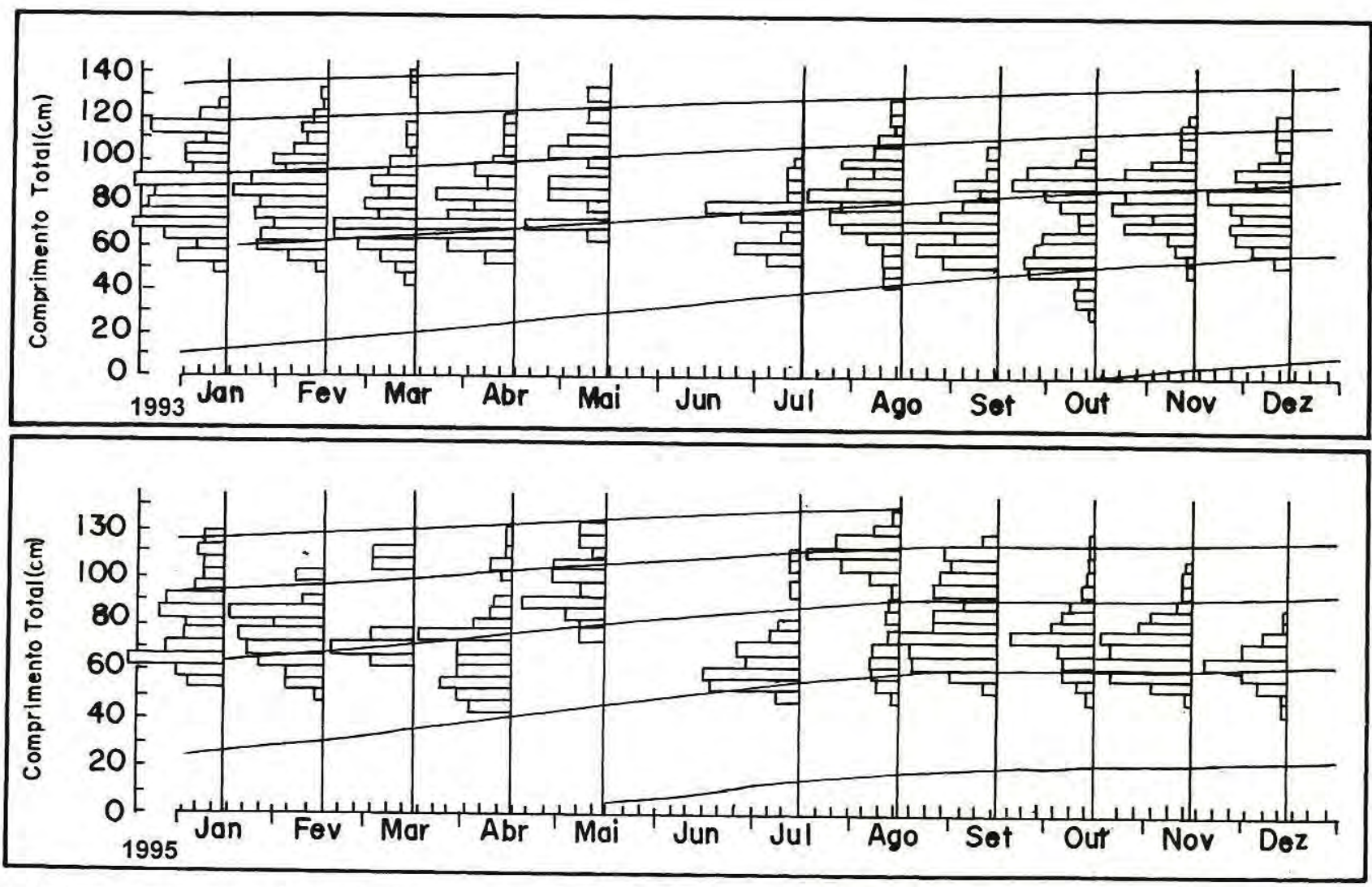

Figura 8. Curvas de crescimento de Pseudoplatystoma tigrinum no Médio Amazonas calculadas com base nos dados de frequências de comprimento 


\section{Taxas de mortalidade}

Usando os comprimentos médios para estimar a mortalidade total de $P$. tigrinum, os valores de $\mathrm{Z}$ obtidos foram 1,22 ano $^{-1}$ e 1,41 ano $^{-1}$ para 1993 e 1995 , respectivamente. As estimativas de $Z$ obtidas através das curvas de captura convertidas em comprimento foram 1,74 ano ${ }^{-1}$ para 1993 e 1,32 ano $^{-1}$ para 1995 (Tab. 5).

As estimativas da mortalidade natural (M) obtidas para 1993 e 1995 foram 0,47 ano $^{-1}$, para ambos os anos, utilizando o valor de $27^{\circ} \mathrm{C}$ para a temperatura da água. Consequentemente, os valores obtidos para a mortalidade por pesca (F) foram 1,01 ano ${ }^{-1}$ para 1993 e 0,90 ano $^{-1}$ para 1995 e, a taxa de explotação $(\mathrm{E})$ de 0,68 ano $^{-1}$ e 0,66 ano $^{-1}$ para 1993 e 1995 , respectivamente (Tab. 3 ).

O comprimento médio em que $50 \%$ dos peixes encontrados pela arte de pesca são retidos $\left(\mathrm{L}_{\mathrm{c}}\right)$ foi estimado $\mathrm{em} 85,0 \mathrm{~cm}$ para 1993 e 86,6 cm para 1995 (Tab. 3).

\section{Rendimento por recruta}

O modelo de rendimento por recruta dá uma indicação do estado atual da pescaria em relação ao rendimento máximo sustentável potencial. Considerando os parâmetros de crescimento e mortalidade estimados e uma taxa de exploração atual de 0,67 ao ano, a curva de rendimento indica que o estoque está sendo explorado muito próximo do máximo rendimento, no seu limite de sustentabilidade.

\section{DISCUSSÃO}

Apesca na Amazônia apresenta forte sazonalidade. A estação seca geralmente é mais produtiva porque os peixes estão migrando e concentrados no canal principal do rio e as capturas são relativamente maiores nessa época. A produção de surubim-tigre mostra que existe considerável variação entre os meses devido às mudanças sazonais e o padrão do CPUE é similar para todos os anos. $\mathrm{Ou}$ seja, apesar dos desvios, as maiores capturas e melhor produtividade da pesca ocorrem na vazante e seca e, conforme as águas vão subindo, os pescadores tendem a gastar mais tempo nas pescarias, deslocando-se para os lagos durante o inverno, quando os rendimentos são muito baixos. As estimativas de CPUE do presente trabalho foram um pouco aquém dos $11,7 \mathrm{~kg} /$ pescador $/$ dia obtidos para $P$. corruscans, no rio São Francisco (Godinho et al.,1997) que foram estimados com base apenas para o período de maior captura.

Em relação ao estudo da dinâmica populacional, no rio Apure, Venezuela, Reid (1983) encontrou que a idade e crescimento de $P$. fasciatum e $P$. tigrinum podiam ser determinados através de anéis anuais das vértebras. Para $P$. tigrinum, as estimativas dos parâmetros de crescimento foram: $\mathrm{L}_{\infty}=146 \mathrm{~cm}$ e $170 \mathrm{~cm}$, para machos e fêmeas, respectivamente, e $\mathrm{k}=0,11$ $\mathrm{ano}^{-1}$ e $0,09 \mathrm{ano}^{-1}$. A longevidade foi estimada entre 25 e 30 anos. Payne (1987) utilizando dados de distribuição de freqüência de comprimentos de $P$. tigrinum do rio Mamoré, Bolívia, e o programa ELEFAN, estimou valores de Lo de 141,8 $\mathrm{cm}, \mathrm{k}$ de 0,12 ano $^{-1}$ e longevidade de 19 anos. Essencialmente, as características dos surubins-tigre dos rios Apure e Mamoré são muito semelhantes àquelas do rio 
Tabela 4. Chave comprimento-idade relativa de Pseudoplatystoma tigrinum estimada, utilizando os parâmetros médios (Tab. 3) e o modelo de crescimento sazonal de von Bertalanffy,

\begin{tabular}{cc}
\hline Idade Relativa (anos) & Comprimento Médio $(\mathrm{cm})$ \\
\hline 0,5 & 28 \\
1,0 & 42 \\
1,5 & 66 \\
2,0 & 76 \\
2,5 & 95 \\
3,0 & 102 \\
3,5 & 116 \\
4,0 & 122 \\
4,5 & 132 \\
5,0 & 136 \\
5,5 & 144 \\
6,0 & 147 \\
6,5 & 153 \\
7,0 & 155 \\
7,5 & 160 \\
8,0 & 162 \\
8,5 & 165 \\
9,0 & 166 \\
9,5 & 168 \\
10,0 & 170 \\
\hline
\end{tabular}

Tabela 5. Parâmetros de entrada e estimativas de mortalidade total (Z) para Pseudoplatystoma tigrinum. (L' representa o tamanho a partir do qual os indivíduos são recrutados à pesca).

\begin{tabular}{lccc}
\hline $\begin{array}{l}\text { Ano da } \\
\text { amostra }\end{array}$ & Parâmetros de entrada & Método & Z \\
\hline 1993 & $L_{\infty}=180 \mathrm{~cm}$ e $L^{\prime}=62,5 \mathrm{~cm}$ & $\begin{array}{c}\text { Comprimento médio } \\
\text { Curva de captura }\end{array}$ & $\begin{array}{c}1,22 \\
1,74\end{array}$ \\
\hline Média & $\mathrm{L}_{\infty}=180 \mathrm{~cm}$ e L'=67,5 cm & $\begin{array}{c}\text { Comprimento médio } \\
\text { Curva de captura }\end{array}$ & 1,41 \\
\hline 1995 & & & 1,32 \\
\hline Média & & & 1,37 \\
\hline
\end{tabular}

Amazonas. A principal diferença é o tamanho máximo; os exemplares do rio Amazonas são maiores. Além disso, no rio Apure parece haver diferenças no crescimento entre machos e fềmeas, com as fêmeas atingindo tamanhos maiores. Em nossas estimativas, a partir do desembarque da pesca comercial, não foi possivel separar machos e fêmeas uma vez que, na maioria das vezes, os peixes são limpos e eviscerados antes dos desembarques. No entanto, o que se observa é que as estimativas da constante de crescimento $(\mathrm{k})$ obtidas para os estoques dos rios Apure e Mamoré são menores que as obtidas no presente trabalho. Tal fato, pode ser um reflexo do tipo de dados e análises 
realizadas. Reid (1983) utilizou intervalos de classe de $1 \mathrm{~cm}$ nos seis dados de marcas nas vértebras e Payne (1987) utilizou apenas 1 mês de dados de distribuição de comprimentos que analisou através do programa ELEFAN, mas também utilizando intervalos de classe de $1 \mathrm{~cm}$.

A mortalidade natural está correlacionada positivamente com as taxas de crescimento. Taxas de crescimento baixo correspondem a mortalidade natural baixa. $\mathrm{O}$ valor de $\mathrm{k}$ de 0,29 ano $^{-1}$ e de $\mathrm{M}$ de 0,47 ano $^{-1}$ definem o surubim-tigre como uma espécie de taxas de crescimento e mortalidade relativamente baixas. Já o valor estimado de mortalidade por pesca $\left(\mathrm{F}=0,95\right.$ ano $\left.^{-1}\right)$ parece ser muito alto $\mathrm{e}$ não apropriado para a estratégia da espécie. Isto está refletido nos resultados do modelo de rendimento por recruta de Beverton \& Holt (1957), o qual indica que o estoque esta sendo explorado no seu limite máximo.

Neste tipo de espécie é freqüente a ocorrência da chamada "sobrepesca de crescimento", na qual ocorre a captura de indivíduos muito pequenos. Nesta situação, a mortalidade por pesca supera a velocidade de crescimento da espécie, impedindo que os exemplares alcancem um tamanho maior, para contribuir de forma mais substancial com os volumes capturados. Para evitar este tipo de sobrepesca deve-se, em primeiro lugar, evitar a captura de individuos muito jovens. A portaria do IBAMA 008/96, de 02/02/ 96 , que estabelece normas gerais para o exercício da pesca na bacia hidrográfica do rio Amazonas, proibe a captura, transporte e comercialização de Pseudoplatystoma tigrinum cujo comprimento total seja in- ferior a $80 \mathrm{~cm}$ (Pinto, 1996).

O tamanho mínimo permitido para a captura é fixado geralmente com base no comprimento no qual os indivíduos se tornam adultos. Entende-se por comprimento médio de primeira maturação gonadal $\left(\mathrm{L}_{50}\right)$ aquele com o qual $50 \%$ dos indivíduos apresentam gônadas em desenvolvimento, ou seja, iniciaram o ciclo reprodutivo. Segundo Vazzoler (1996), o conhecimento desse parâmetro populacional é de importância fundamental para a administração racional dos estoques. Porém, o início da maturação gonadal de $P$. tigrinum na Amazônia brasileira ainda não foi devidamente estudada, apenas Isaac $\mathrm{et} \mathrm{al}$. (no prelo) estimaram um $\mathrm{L}_{\text {min }}$ de $52 \mathrm{~cm}$ para ambos os sexos. Gil \& Martinez (1995), estudando exemplares de $P$. tigrinum na Colômbia, estimaram um $\mathrm{L}_{50}$ de 67 e $102 \mathrm{~cm}$, para machos e fêmeas de $P$. tigrinum, respectivamente. Para $P$. fasciatum, os mesmos autores estimaram um $\mathrm{L}_{50}$ de $60 \mathrm{~cm}$ para machos e $83 \mathrm{~cm}$ para fềmeas, enquanto que Valderrama (1988) estimou um $\mathrm{L}_{50}$ de 79 $\mathrm{cm}$ para ambos sexos. Considerando estes valores e os resultados da análise de rendimento por recruta do presente trabalho parece que a proibição da captura de exemplares menores do que $80 \mathrm{~cm}$ é uma medida prudente e correta. Segundo a curva de crescimento, este tamanho corresponde a indivíduos de mais de dois anos.

Apesar de que as pescarias do Médio Amazonas são multiespecíficas e utilizam diversos tipos de aparelhos, de acordo a Isaac et al. (1993), o estabelecimento de um tamanho mínimo de captura é um instrumento que pode 
ser considerado adequado para a região. Observando-se a dinâmica das pescarias artesanais na Amazônia, fica bastante evidente que, de maneira geral, os pescadores possuem técnicas dirigidas a determinadas espécies, evitando a captura de espécies ou tamanhos indesejados. Além disso, a regulamentação que estabelece o tamanho mínimo de captura é passível de controle, o qual pode ser facilmente realizado no momento do desembarque. Já a proibição de um tipo especifico de aparelho ou de pesca, é praticamente incontrolável, considerando a distância dos pesqueiros entre si.

\section{AGRADECIMENTOS}

Os autores agradecem a todos os coletores e digitadores de dados biológicos e de desembarque pesqueiro do Projeto IARA, ao IBAMA, GTZ e PTU/CNPq pelo aporte financeiro.

\section{Bibliografia citada}

Barthem, R.B.; Goulding, M. 1997. Os bagres balizadores: ecologia, migração e conservação de peixes amazônicos, SCM/MCTCNPq/IPAAM. $140 \mathrm{p}$.

Beverton, R.J.H.; Holt, S.J. 1956. A review of methods for estimating mortality rates in exploited fish populations, with special reference to sources of bias in catch sampling. Rapports et Procès-Verbaux.des Réunions Conseil International pour $L^{\prime}$ Exploration de la Mer 140 (1): 67-83.

..... 1957. On the dynamics of exploited fish populations. Fish Invest., Ser. II, 19: 533 p.

Coy, Y.S. 1994. Aspectos de la biologia pesquera de las poblaciones de los grandes bagres (Ostariophysi: Siluriformes, Pimelodidae) en el sector colombiano del rio Amazonas. Trabalho de Conclusão de Curso de Licenciatura em Biologia, Santafé de Bogotá, 156p.
Ferreira, E.J.G; Zuanon, J.A.S. \& Santos, G.M. 1998. Peixes Comerciais do Médio Amazonas: Região de Santarém, Pará. IBAMA. Coleção Meio Ambiente. Série Estudos Pesca, (18): 214 p.

Gayanilo, F.C., Jr.; Sparre, P. and Pauly, D. 1994. The FAO-ICLARM Stock Assessment Tools (FISAT) User's Guide. FAO Computerized Information Series (Fisheries) (6): $186 \mathrm{p}$.

Gil, H.R.; Martinez, R.E.A. 1995. El bagre rayado Pseudoplatystoma fasciatum (Linnaeus) y Pseudoplatystoma tigrinum (Valenciennes): aspectos biologico pesquero en el alto rio Meta. Boletim Cientifico INPA, (3):157-167.

Godinho, H.P.; Tallarico de Miranda, M.O.; Godinho, A.L.; Santos, J.E. 1997. Pesca e biologia do surubim Pseudoplatystoma corruscans no rio São Francisco. In: Miranda, M.O.T. (Org.). Surubim. IBAMA. Coleção Meio Ambiente. Série Estudos Pesca, 19: 27-42.

Goulding, M. 1979. Ecologia da Pesca do Rio Madeira. INPA, Manaus, $172 \mathrm{p}$.

1980. The Fishes and the Forest. Explorations in Amazonian Natural History. University of California Press, Berkeley, CA, 280 p.

IBAMA. 1995. Projeto IARA - Administração dos Recursos Pesqueiros do Médio Amazonas: Estados do Pará e Amazonas. IBAMA. Coleção Meio Ambiente. Série Estudos Pesca, (15): 100 p.

Isaac, V.J.; Rocha, V.L.C.; Mota, S.Q.C. (no prelo). Ciclo reprodutivo de algumas espécies de peixes de valor comercial do Baixo Amazonas. IBAMA. Coleção Meio Ambiente. Série Estudos Pesca.

; Ruffino, M.L. (no prelo). A Estatística Pesqueira no Baixo Amazonas: Experiência do Projeto IARA. IBAMA. Coleção Meio Ambiente. Série Estudos Pesca.

Pauly, D. 1980. On the interrelationships between natural mortality, growth parameters and mean environmental temperature in 175 fish stocks. International Council for the Exploration of the Sea, 39 (2): 175-192.

1984. Fish population dynamics in tropical waters: A manual fou use with pro- 
grammable calculators. ICLARM Studies and Reviews (Philippines), 8: $325 \mathrm{p}$.

; David, N. 1981. Elefan I, a Basic program for the objective extraction of growth parameters from length-frequency data. Meeresforsch 28 (4):205-211.

; Munro, J.L. 1984. Once more on the comparison of growth in fish and invertebrates. Fishbyte 2 (1): 21 .

Payne, A.I. 1987. A preliminary stock assessment survey of the fishery at Trinidad in the Rio Marmoré. Report for ODA Consultants, $34 \mathrm{p}$ (mimeo).

Petrere Jr., M. 1992. Pesca na Amazônia. p. 72-78. In: Secretaria de Estado de Ciência, Tecnologia e Meio Ambiente-PARÁ. SIMDAMAZÔNIA, Seminário Internacional Sobre Meio Ambiente, Pobreza e Desenvolvimento da Amazônia. Anais: Belém. PRODEPA. 567 p.

Pinto, W.D. 1996. Legislação Federal de Meio Ambiente. IBAMA, Brasília. 3 volumes, $2083 \mathrm{p}$.

Reid, S. 1983. La biologia de los bagres rayados Pseudoplatystoma fasciatum y $P$. tigrinum en la cuenca del rio Apure, Venezuela. Revista UNELLEZ de Ciência y Technologia, 1:13-41.

Ruffino, M.L.; Isaac, V.J. 1994. The Fisheries of the Lower Amazon: Questions of Management and Development. Acta Biologica Venezuelica, 15 (2):37-46.
; Isaac, V.J. 1995. Life Cycle and Biological Parameters of Several Amazon Fish Species. NAGA, The ICLARM Quarterly, 18(4):41-45.

Santos, G.M. 1987 Composição do pescado e situação da pesca no Estado de Rondônia. Acta Amazonica, 16/17, supl.:43-84.

Smith, N.J.H. 1981. Mu.., Fishes and the Amazon. Columbia University Press, New York. $180 \mathrm{p}$.

Sparre, P.; Ursin, E.; Venema, S.C. 1992. Introduction to Tropical Fish Stock Assessment. Part 1-Manual. FAO Fisheries Technical Paper (306/1): $337 \mathrm{p}$.

Valderrama, M.B.; Zarate, V.M.; Vera, G.V.; Moreno, Z.C.; Caraballo, P.G.; Martinez, J.R. 1988. Determinación de la talla media de madurez y analisis de la problematica con referencia a las tallas medias de captura del bagre rayado (Pseudoplatystoma fasciatum) Linnaeus 1766 (Pisces: Pimelodidade) en la cuenca del rio Magdalena, Colombia. TRIANEA (Act. Cient. Tecn. INDERENA), 2:537-549.

Vazzoler, A.E.A. de M. 1996. Biologia da reprodução de peixes teleósteos: teoria e prática. EDUEM, São Paulo. 169p. 